\title{
Autorinnen und Autoren | The Authors
}

Christof Aichner studierte Geschichte an den Universitäten Innsbruck und Zürich und promovierte im Jahr 2015. Seither war er Mitarbeiter in mehreren Projekten zur österreichischen Universitäts- und Wissenschaftsgeschichte, unter anderem der digitalen Edition der Korrespondenz von Leo Thun-Hohenstein. Er ist Sekretär der Kommission für Neuere Geschichte Österreichs (Innsbruck/Wien).

Matthias Berg war seit 2007 wissenschaftlicher Mitarbeiter am Institut für Geschichtswissenschaften der Humboldt-Universität zu Berlin, wo er 2013 promoviert wurde. Seine Forschungsschwerpunkte liegen in der Historiographie- und Wissenschaftsgeschichte, er ist Mitverfasser von „Die versammelte Zunft. Historikerverband und Historikertage in Deutschland 18932000“ (Göttingen 2018). Seit 2019 ist er wissenschaftlicher Mitarbeiter der Historischen Kommission bei der Bayerischen Akademie der Wissenschaften.

Beate Ceranski, Wissenschaftshistorikerin (Staatsexamen Physik und Mathematik Sek. II/I an der Universität Bonn, Promotion in Geschichte der Naturwissenschaften an der Universität Hamburg, Habilitation für Geschichte der Naturwissenschaften und Technik an der Universität Stuttgart), forscht und lehrt an der Abteilung für Geschichte der Naturwissenschaften und Technik am Historischen Institut der Universität Stuttgart. Sie arbeitet unter anderem zur Frauen- und Geschlechtergeschichte der Naturwissenschaften, zur Geschichte der Radioaktivitätsforschung, zur Hochschul- und Sammlungsgeschichte.

Ning de Coninck-Smith is professor of the history of education at Aarhus University. Before joining Aarhus University in 2002, she was employed at the University of Southern Denmark where she defended her habilitation in 2000. She has written extensively on the history of education and childhood and is currently chairing a research project on gendered and affective readings of the history of Aarhus University. Ning de Coninck-Smith is co-editor of a Cultural History of higher learning, to be published by Bloomsbury in 2024.

Pieter Dhondt is senior lecturer in General History and head of the Department of Geographical and Historical Studies at the University of Eastern Finland. He published extensively on the intercultural transfer of university ideas within Europe in the nineteenth century, the history of academic mobility, student revolts and university celebrations. His current research focuses primarily on the history of (dealing with) medical uncertainty in medical training.

Edith Glaser ist seit 2005 Professorin für Historische Bildungsforschung an der Universität Kassel. Sie wurde mit einer Arbeit über die Anfänge des Frauenstudiums an der Universität Tübingen promoviert. Die Habilitation erfolgte an der Universität Halle-Wittenberg mit einer professionsgeschichtlichen Arbeit über den Lehrerinnenberuf. Zu ihren Forschungsschwerpunkten gehören u.a. die pädagogisch-historische Geschlechterforschung, Analysen von Bildungsreformprozessen und die Disziplingeschichte der Erziehungswissenschaft. 
Martin Göllnitz hat Geschichte und Germanistik an der Universität Kiel studiert. Von 2014 bis 2017 war er dort wissenschaftlicher Mitarbeiter und wurde mit einer Studie über jungakademische NS-Funktionäre promoviert. Im Anschluss forschte er an der Universität Odense und von 2017 bis 2019 am Arbeitsbereich Zeitgeschichte an der Universität Mainz. Seit März 2019 ist er als wissenschaftlicher Assistent an der Professur für Hessische Landesgeschichte der Universität Marburg tätig.

Anton F. Guhl, Studium in Hamburg und Galway, Berenberg-Preis für Wissenschaftssprache 2015, Promotion als Stipendiat der Studienstiftung 2017 an der Universität Hamburg zur Entnazifizierung der Universität (DGGMNT-Förderpreis 2017), 2017- 2020 Akademischer Mitarbeiter am Department für Geschichte (ITZ) am KIT, seit 2021 Projektmitarbeiter im KIT-Archiv für die Konzeption und Realisierung der für 2025 geplanten Ausstellung „100 Objekte zur Geschichte des KIT“.

Gisela Hürlimann wurde an der Universität Zürich promoviert und an der Universität Freiburg (Schweiz) habilitiert. Sie ist seit September 2021 Professorin für Technik- und Wirtschaftsgeschichte an der TU Dresden. Zu ihren Schwerpunkten zählen die Geschichte der Mobilität, öffentlicher Unternehmen, Infrastrukturen und Finanzen, von Rohstoffen und Mensch-Nutztierbeziehungen.

Alexander Kather studierte zwischen 2013 und 2019 Geschichtswissenschaft, Romanistik und Bildungswissenschaften an den Universitäten Kassel und Straßburg. Seit 2020 arbeitet er als wissenschaftlicher Mitarbeiter im Fachgebiet Historische Bildungsforschung an der Universität Kassel. Neben universitätsgeschichtlichen Themen gilt sein Interesse der Geschichte des Fremdsprachenunterrichts und der Fremdsprachendidaktik.

Sarah Kramer studierte Geschichtswissenschaften, Germanistik, Lateinische Philologie und Erziehungswissenschaften an der Philipps-Universität Marburg sowie dem University College Cork (Irland). Zurzeit promoviert sie als Stipendiatin des Marburger Arbeitskreises für Universitätsgeschichte zu Hochschulprotesten, Konfliktdynamiken und Bedrohungskommunikation an der Universität Marburg (1960 bis 1980) und arbeitet als Wissenschaftliche Hilfskraft am SFB/ TRR 138 „Dynamiken der Sicherheit“.

Verena Kümmel studierte Geschichte, Kunstgeschichte und Wirtschaftswissenschaften an der TU Darmstadt. Nach Stationen in Münster, Darmstadt und Mainz promovierte sie an der Universität Duisburg-Essen mit einer Arbeit über den Umgang mit den Leichen von Philippe Pétain und Benito Mussolini. Von 2018 bis 2020 hat sie das Forschungsprojekt zu Waldtraut Krützfeldt-Eckhard als erster Rektorin der Evangelischen Fachhochschule Darmstadt an der heutigen Evangelischen Hochschule geleitet. Der Abschlussbericht befindet sich in Vorbereitung.

Catherine Maurer ist Professorin für Geschichte des 19. und 20. Jahrhunderts mit Schwerpunkt auf Deutsche Geschichte an der Universität Straßburg. Ihre wichtigsten Publikationen sind: Der Caritasverband zwischen Kaiserreich und Weimarer Republik. Zur Sozial- und Mentalitätsgeschichte des caritativen Katholizismus in Deutschland (Freiburg im Breisgau, Lambertus, 2008), Une université nazie sur le sol français. Nouvelles recherches sur la Reichsuniversität de Strasbourg (Revue d'Allemagne, 2011) und: La ville charitable. Les œuvres sociales catholiques en France et en Allemagne (Paris, Éditions du Cerf, 2012). 
Juliane Mikoletzky studierte Geschichte und Germanistik an der Ruhr-Universität Bochum und promovierte 1986. Daneben war sie in verschiedenen Projekten, als Universitätsassistentin und nach ihrer Übersiedlung nach Wien als Lektorin an der Universität Wien tätig. Seit 1992 arbeitet sie als wissenschaftliche Mitarbeiterin am Archiv der TU Wien, 2001-2015 als Leiterin, seither als freie Mitarbeiterin. Sie forscht zu Themen der Wirtschafts-, Sozial-, Technik- und Wissenschaftsgeschichte des 19. und 20. Jahrhunderts.

Paula Riling studiert European Cultures and Society an der Universität Flensburg. Im Rahmen ihres Studiums befasst sie sich vor allem mit dem Phänomen des „Othering“ in der Europäischen Union und mit Kulturgeschichte.

Vivian Yurdakul studierte Deutsche Literatur und Geschichte an der Humboldt-Universität zu Berlin sowie Geschichte und Kultur der Wissenschaft und Technik an der Technischen Universität Berlin. Seine Forschungsschwerpunkte sind die Verflechtung von Wissenschaften und Recht im Nationalsozialismus sowie die Wissenschafts- und Verwaltungsgeschichte des „Dritten Reiches“. Er ist Wissenschaftlicher Mitarbeiter an der Humboldt-Universität zu Berlin sowie an der Bergischen Universität Wuppertal.

Gunnar B. Zimmermann, bis 2020 Wissenschaftlicher Mitarbeiter der Arbeitsstelle für Universitätsgeschichte an der Universität Hamburg und Mitherausgeber einer mehrbändigen Universitätsgeschichte; Dissertation über „Bürgerliche Geschichtswelten im Nationalsozialismus. Der Verein für Hamburgische Geschichte zwischen Beharrung und Selbstmobilisierung“ (2019); Forschungen zur Bildungs-, Universitäts- und Wissenschaftsgeschichte sowie zur Bürgertums- und Demokratiegeschichte. 
